\title{
Recent progress of nuclear data measurement in Japan
}

\author{
M. Baba \\ Cyclotron and Radioisotope Center, Tohoku University, Sendai 980-8578, Japan
}

\begin{abstract}
Recent progress of nuclear data measurement in Japan is reviewed briefly. Experiments are conducted over a wide energy region from low energy to intermediate and high energy regions including charged-particle induced reactions. Experimental work is described together with facilities and apparatus which were used in the experiments emphasizing the recent progress.
\end{abstract}

\section{Introduction}

Experimental data play a crucial role in nuclear data evaluation. Recent advances in nuclear energy programs and the extension of radiation utilization lead to the requirement of nuclear data in new areas like minor actinides and high energy region in addition to the refinement of traditional longstanding data. To reply to these requirements, development of facilities and apparatuses, and new experiments employing them are promoted. In Japan, various kinds of nuclear data measurement and technology development are conducted for detector and data acquisition systems and neutron sources to enable new quality data.

The present paper provides a brief review on recent progress of nuclear data measurement in Japan categorizing into 1) capture and fission, 2) neutron production, 3) chargedparticle production reactions and 4) neutron source.

\section{Capture and fission cross sections}

Neutron induced capture cross sections are of prime importance in the nuclear energy program and also in basic fields like nuclear synthesis calculation. In Japan, studies of neutron capture cross section are conducted very actively in several laboratories and inter-laboratory collaboration.

\subsection{Japan Atomic Energy Agency (JAEA)}

The JAEA group has conducted neutron induced capture cross section measurements in thermal and low energy region using neutron beam from reactors and an electron linac [1]. Detectors are high efficiency Ge detectors coupled with data acquisition system employing a digital signal processing technique (DSP). They reported a number of data on cross sections, branching ration, and resonance integrals of minor actinides (MA) and long-lived fission products (LLFP) which are of interest from a view point of nuclear transmutation.

They are working too as main members in the Nuclear Data Project in Japan which is mentioned in section 2.2.

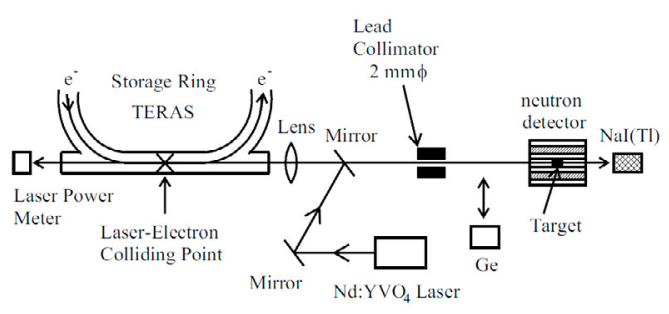

Fig. 1. Schematic view of Laser-Compton system [2].

The group has also been conducting the inverse $(\gamma, \mathrm{n})$ experiment to obtain neutron capture cross section data using a "monoenergetic" photon beam provided by a laser-Compton scattering facility at AIST (Advanced Institute for Science and Technology) shown in figure 1 [2,3]. A similar facility is used also in JAEA to study neutron capture cross sections of fission product in collaboration with university groups [4].

\subsection{Tokyo Institute Technology (TIT) group}

The TIT group has conducted a series of measurements of neutron capture cross sections and $\gamma$-ray spectrum for light to medium heavy elements which are of interest for nucleosynthesis, and for transmutation of LLFP and MA [5,6]. Measurements are done using monoenergetic or continuous spectrum neutrons produced by the ${ }^{7} \mathrm{Li}(\mathrm{p}, \mathrm{n})^{7} \mathrm{Be}$ reactions with a pulsed proton beam from a $3 \mathrm{MV}$ Peletron. A large $\mathrm{NaI}(\mathrm{Tl})$ $\gamma$-ray detector with an anti-Compton suppressor of BGO is used as high efficiency $\gamma$-ray detector. They reported a number of experimental data for various nuclides. The group has worked as principal members of The Nuclear Data Project-1 in Japan.

\subsection{Kyoto University, Research Reactor Institute}

In Kyoto University Research Reactor Institute (KURRI), a $46 \mathrm{MeV}$ electron linac is used for experiments with a TOF 


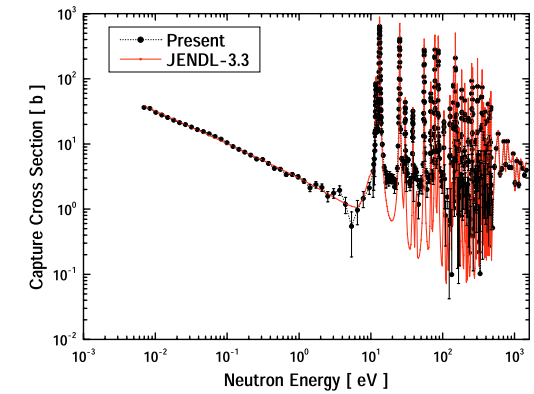

Fig. 2. ${ }^{205} \mathrm{Pd}(\mathrm{n}, \gamma)$ data at Kyoto Univeristy [7].

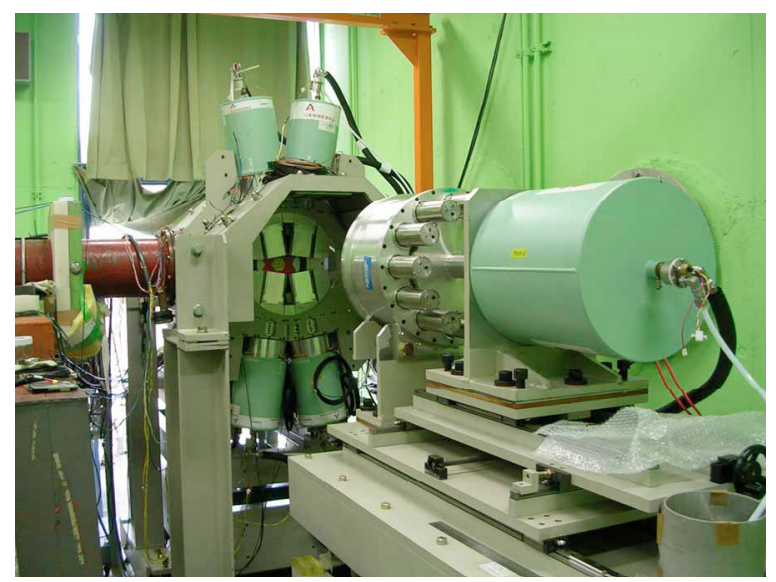

Fig. 3. $4 \pi$ Ge $\gamma$-ray detector setup at KURRI [8-11].

method and a lead-slowing down spectrometer (KULS). The group is measuring neutron capture cross sections of LLFP using a continuous neutron beam and a $4 \pi$ BGO $\gamma$-ray detector. An example of the measurement is shown in figure 2 . The beam line is also used for test of a $4 \pi$ germanium detector in the Nuclear Data Project.

\subsection{Nuclear Data Project -1,-2}

The project aims the development of $4 \pi \mathrm{Ge}$ detector to enable cascade $\gamma$-ray measurement in Ge matrix configuration (phase-1, led by Dr M. Igashira), and measurement of capture and fission cross sections of MA and LLFP using the detector (phase-2, led by Dr Y. Kiyanagi). The detector and a high speed data acquisition system have been developed and are now almost complete [8-10]. Preliminary data of ${ }^{237} \mathrm{~Np}(\mathrm{n}, \gamma)$ obtained at KURRI are shown in figure 4 [11]. They will be used in the measurement at the intense pulsed neutron source J-PARC (Japan Proton Accelerator Research Complex, see sect. 5) which will be operational in 2008, for measurement of MA ad LLFP in collaboration with other project research.

Under the project, fission cross section measurement of MA was also undertaken using KULS and DSP to enhance the energy range. The example of the fission cross section results is shown in figure 5 [12].

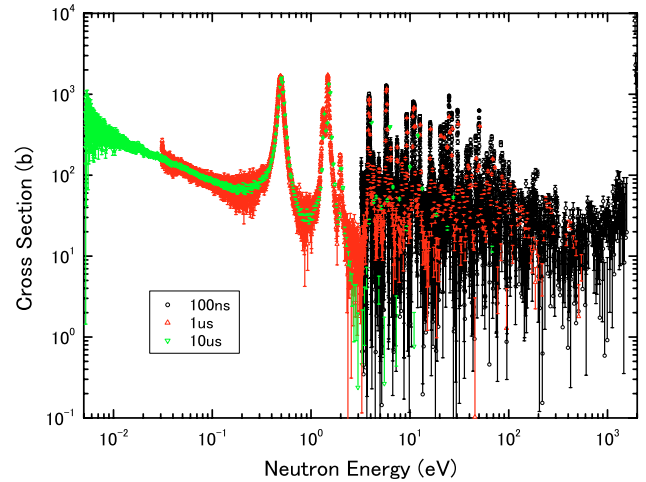

Fig. 4. Preliminary data of ${ }^{237} \mathrm{~Np}(\mathrm{n}, \gamma)$ by the nuclear data project [11].

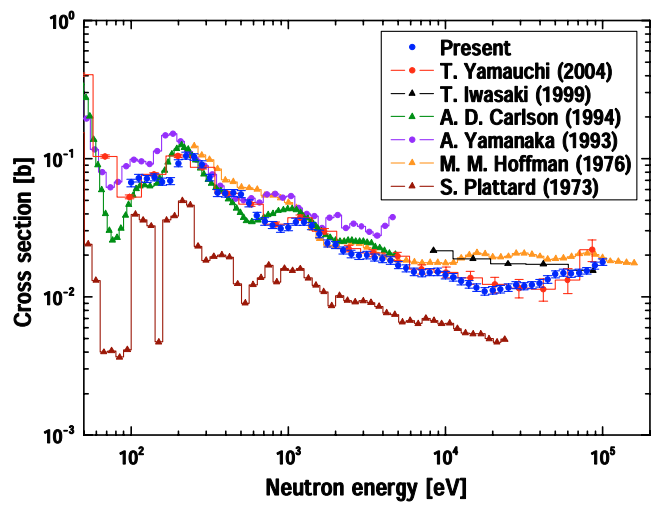

Fig. 5. Fission cross section data at Kyoto University Lead Slowing down spectrometers [12].

\section{Neutron production}

Neutron production cross sections and thick target neutron yields (TTY) are of prime importance in shielding design of accelerator facilities. They have been studied at Cyclotron \& Radioisotope Center (CYRIC), Tohoku University, RCNP (Research Center for Nuclear Physics) of Osaka University, and by Kyushu University group (Ishibashi et al.) at the spallation neutron source LANSCE (Los Alamos Neutron Scattering Center).

\subsection{Tohoku University, Cyclotron}

At CYRIC, Tohoku University, measurement have been done using a K-110 MeV cyclotron equipped with a beam swinger, beam chopper and a well collimated flight path for (p,n) and $(\mathrm{d}, \mathrm{n})$ reactions up to $70 \mathrm{MeV}$ protons and $40 \mathrm{MeV}$ deuterons, respectively $[13,14]$. The deuteron data provided a data base for the design of IFMIF (International Fusion Material Irradiation test Facility). Figure 6 illustrates an example of TTY data for $\mathrm{Li}(\mathrm{d}, \mathrm{n})$ reaction at $40 \mathrm{MeV}$. The data cover the almost entire range of the spectrum and clarify the spectrum shape with good energy resolution. The data provide basis for the design of IFMIF and the evaluation of radiation damage in the irradiation cell. Figure 7 shows the angle dependent neutron spectrum of the ${ }^{7} \mathrm{Li}(\mathrm{p}, \mathrm{n})$ reaction (thin target corresponding 


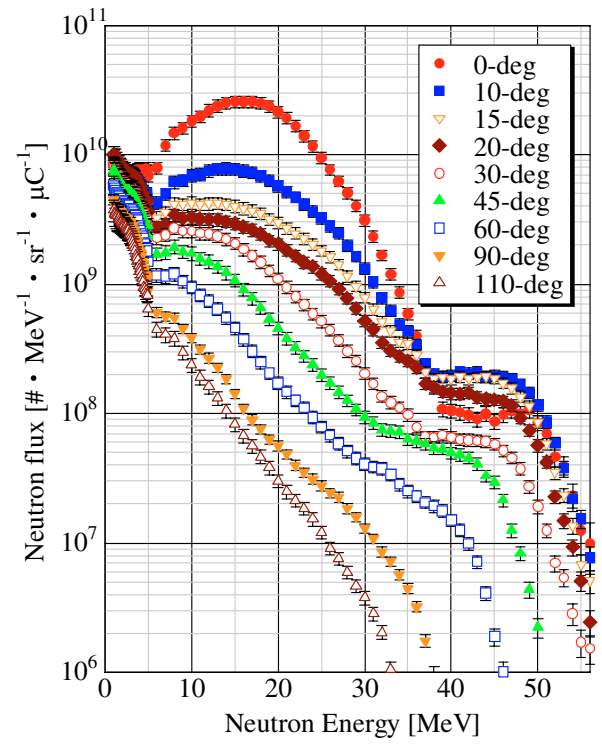

Fig. 6. Thick target neutron yield for $\mathrm{Li}(\mathrm{d}, \mathrm{n})$ at $40 \mathrm{MeV}$ [13].

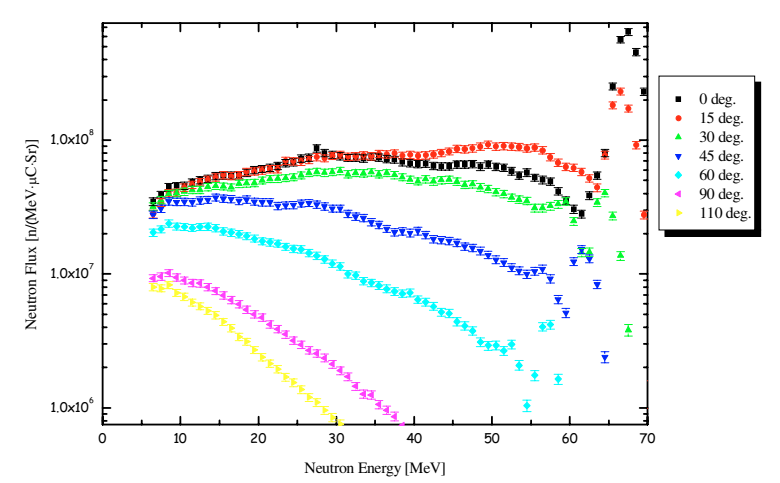

Fig. 7. Angle-dependent neutron emission spectra of ${ }^{7} \mathrm{Li}(\mathrm{p}, \mathrm{n})$ reaction [14] at $7 \mathrm{MeV}$.

to $\sim 2 \mathrm{MeV}$ loss) which is one of the major neutron sources above $20 \mathrm{MeV}$.

\subsection{RCNP, Osaka Univ.}

The experiments at RCNP, Osaka University were carried out by the group of JAEA and universities. Measurements were done for thick targets of $\mathrm{C}, \mathrm{Al}$ and $\mathrm{Fe}$ at 250 and $350 \mathrm{MeV}$ using a proton beam from a $400 \mathrm{MeV}$ ring cyclotron and a conventional TOF method [15]. An example of experimental data is shown in figure $8[15,16]$ in comparison with theoretical calculation. Measurements were also done for the neutron transmission of shielding materials to provide shielding benchmark data.

\section{$3.3(p, n)$ spectrum measurements at LANSCE}

Figure 9 illustrates the schematic view of the experimental setup for neutron emission measurement at LANSCE. A collimated neutron beam from the LANSCE/WNR target 4 is incident on scattering samples placed at the center of detector

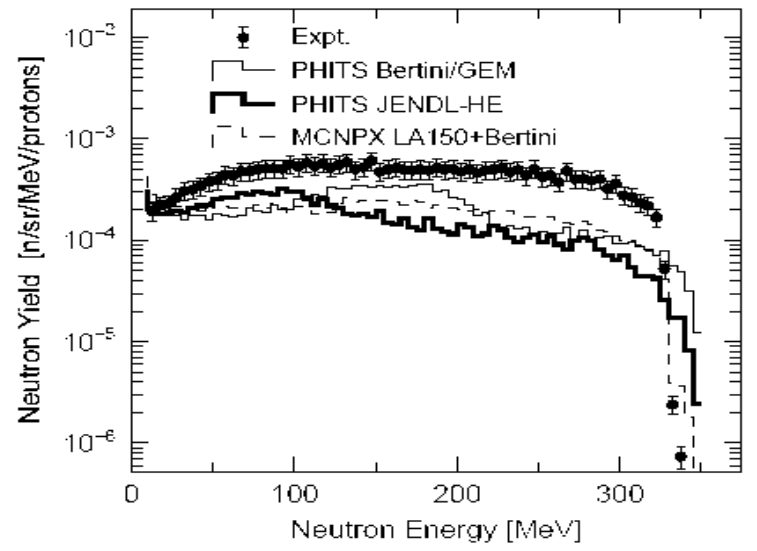

Fig. 8. TTY for $\mathrm{Fe}(\mathrm{p}, \mathrm{xn})$ reaction at $392 \mathrm{MeV}[15,16]$.

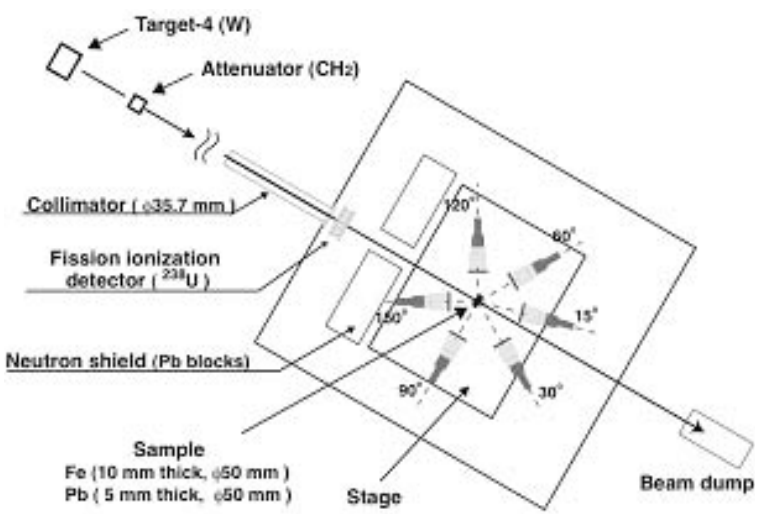

Fig. 9. Experimental setup for neutron emission spectrum measurement at LANSCE [17].

array. Neutrons emitted are detected by NE213 detector and the information on TOF and the pulse height is recorded. The TOF provides the energy of incident neutrons and the pulse-height data the energy spectrum of emitted neutrons through an unfolding procedure of the pulse-height data. The unfolding is carried out using response functions measured by the detectors in the same source. Figure 10 illustrates an example of the unfolded spectrum of the $\mathrm{Fe}(\mathrm{n}, \mathrm{xn})$ reaction for $150 \mathrm{MeV}$ neutrons which were obtained using a moving source model as an initial guess of the continuum spectrum (fig. 11). The data are still preliminary but the method looks promising to obtain new data for such an unexplored energy region.

They are also developing a new spectrometer of phoswitch type for secondary neutrons which will provide the emission spectrum directly without unfolding procedure.

\section{Charged particle emission}

Charged particle emission reactions cause radiation effects in materials and also are the origin of radiation dose by neutrons and protons. In the case fusion reactor, they are also important as reactions for breeding the fuel. For assessment of these effects, energy-angle distributions of charged particles are required to evaluate energy deposit by these particles. 


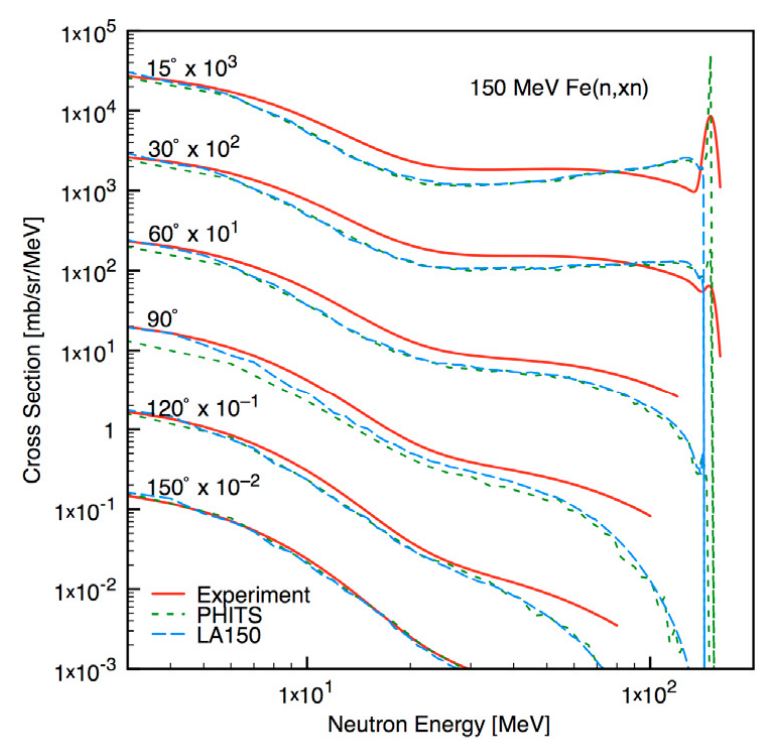

Fig. 10. Results of neutron emission spectra for $\mathrm{Fe}(\mathrm{p}, \mathrm{xn})$ reaction at $150 \mathrm{MeV}$ [17].

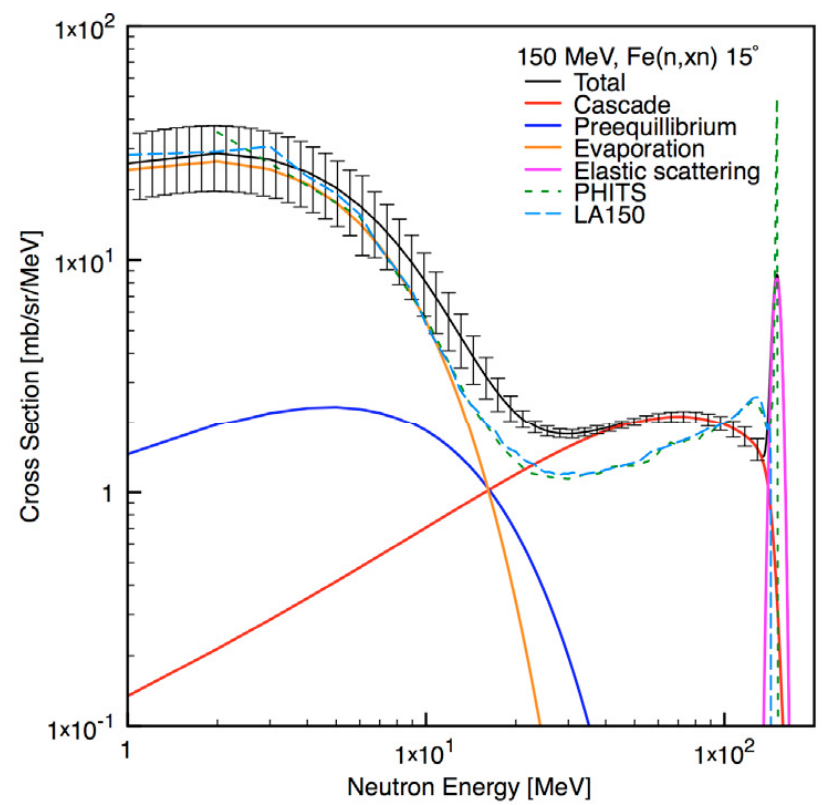

Fig. 11. Moving source model for neutron emission spectrum.

In Japan, three groups have conducted charged particle emission reactions: 1) Osaka University-JAEA FNS group at $14 \mathrm{MeV}$, 2) Kyushu University group (Uozumi et al.) at intermediate energy region, and 3) Production of fragments (particles heavier than lithium) by Tohoku University - KEK group.

\subsection{Experiment for $14 \mathrm{MeV}$ neutrons}

In Osaka University JAEA FNS collaboration, they have conducted experiment using a well collimated pencil beam

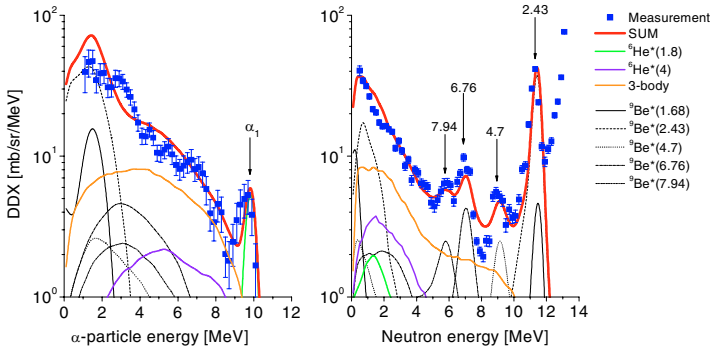

Fig. 12. Analysis of ${ }^{9} \mathrm{Be}(\mathrm{nx} \alpha)$ reaction at $14 \mathrm{MeV}$ [18].

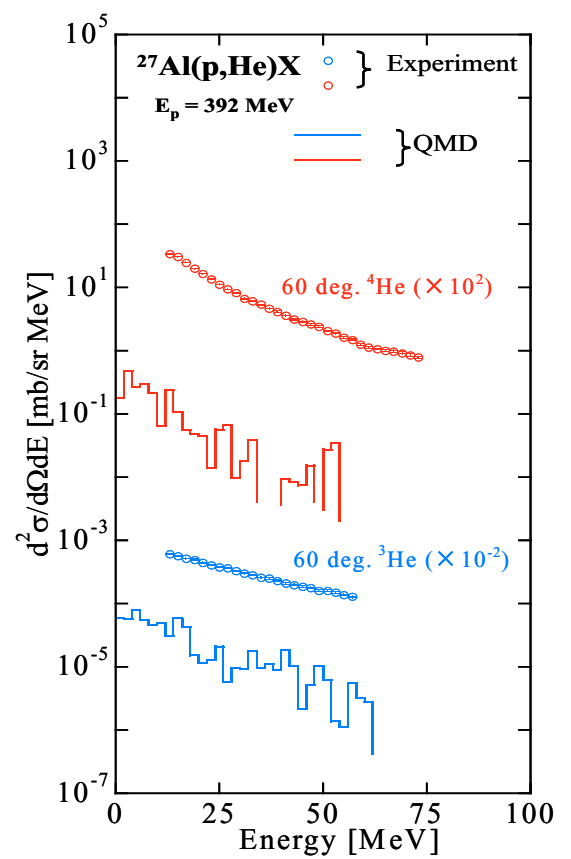

Fig. 13. Differential cross sections for ${ }^{27} \mathrm{Al}\left(\mathrm{p},{ }^{3} \mathrm{He}\right),\left(\mathrm{p},{ }^{4} \mathrm{He}\right)$ reactions at $392 \mathrm{MeV}$ [20].

of $14 \mathrm{MeV}$ provided by an intense $14 \mathrm{MeV}$ source FNS (Fusion Neutronics Source) at JAEA, Tokai $[18,19]$. The pencil beam provides very a good environment for detection of secondary charged-particle and neutrons because detectors are not irradiated by direct neutrons. Taking the advantage, they have conducted measurement of 1) double - differential charged particle emission reactions [18], and 2) correlation measurement of two neutrons in $(n, 2 n)$ reactions [19]. Figure 12 illustrates an example of $\alpha$-particle spectra from Be bombarded by $14 \mathrm{MeV}$ neutrons, which was obtained by using counter-telescope detectors with low threshold. The data exhibits complex spectrum shape and strong angular dependence reflecting the fact that Be has various decay mode including sequential and simultaneous breakup processes resulting in two-neutron emission. The experimental data are compared with calculation based on the Monte Carlo method assuming sequential decay with various branching. Parameters are adjusted to fit the experimental data. The calculation reproduces the measurement fairly well and may be used to predict neutron emission spectrum as well as charged-particle spectrum. 


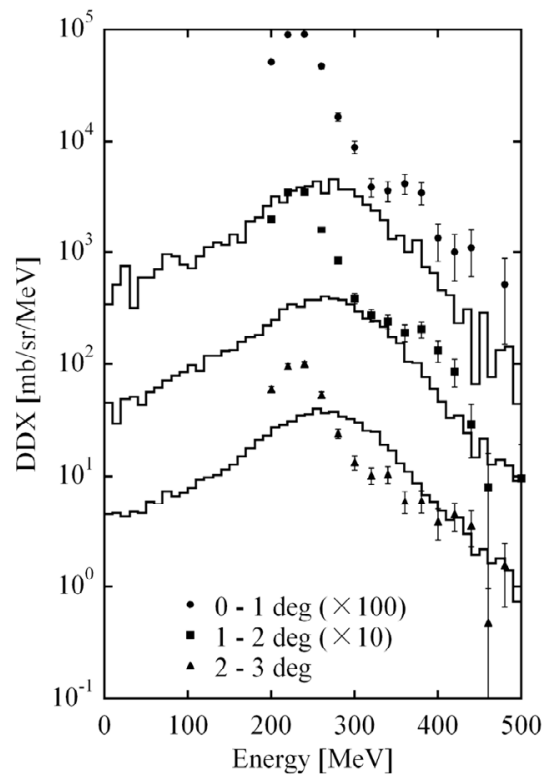

Fig. 14. Proton emission spectra from the ${ }^{12} \mathrm{C}+{ }^{12} \mathrm{C}$ reaction at $290 \mathrm{MeV} / \mathrm{c}$. The solid line shows QMD calculation $[18,19]$.

\subsection{Intermediate energy region}

Kyushu University group has conducted a series of measurement of double-differential charged-particle emission data in the intermediate energy region [20,21]. The experiment at RCNP is induced by $392 \mathrm{MeV}$ protons, and charged particles are detected and identified using counter-telescopes consisting of GSO stacks to stop energetic particles produced in the reaction. Figure 13 illustrates typical data for ${ }^{3} \mathrm{He}$ and ${ }^{4} \mathrm{He}$ from Al. The results are compared with theoretical calculations using QMD (Quantum Molecular Dynamics). The calculation greatly underestimates the experimental emission spectra while the shape of spectrum look similar. It is found that the differences can be reduced by use of an advanced AMD (Anti-symmetrized Molecular Dynamics) code.

Similar comparison is shown in figure 14 which is the proton spectrum for the ${ }^{12} \mathrm{C}+{ }^{12} \mathrm{C}$ reaction at $290 \mathrm{MeV} / \mathrm{c}$ measured at HIMAC (Heavy Ion Medical Accelerator in Chiba). The group is now developing an advanced detector system which can be applied to higher energy particles by combining energy measurement and TOF measurement. The TOF is used for energetic particles which penetrate the energy detector.

\subsection{Fragment production}

Heavy secondary charged particles are considered to be a cause of nucleon-induced single event upset of semiconductor devices. For the analysis of the phenomena, energy angular distribution data are required not only for light ones but also for fragments. However, differential cross section data for fragment production are practically non-existing. For fragment detection, special techniques are required because of very large stopping power and relatively smaller yield. The Tohoku University and KEK (High energy accelerator organization) group developed a detector for differential fragment
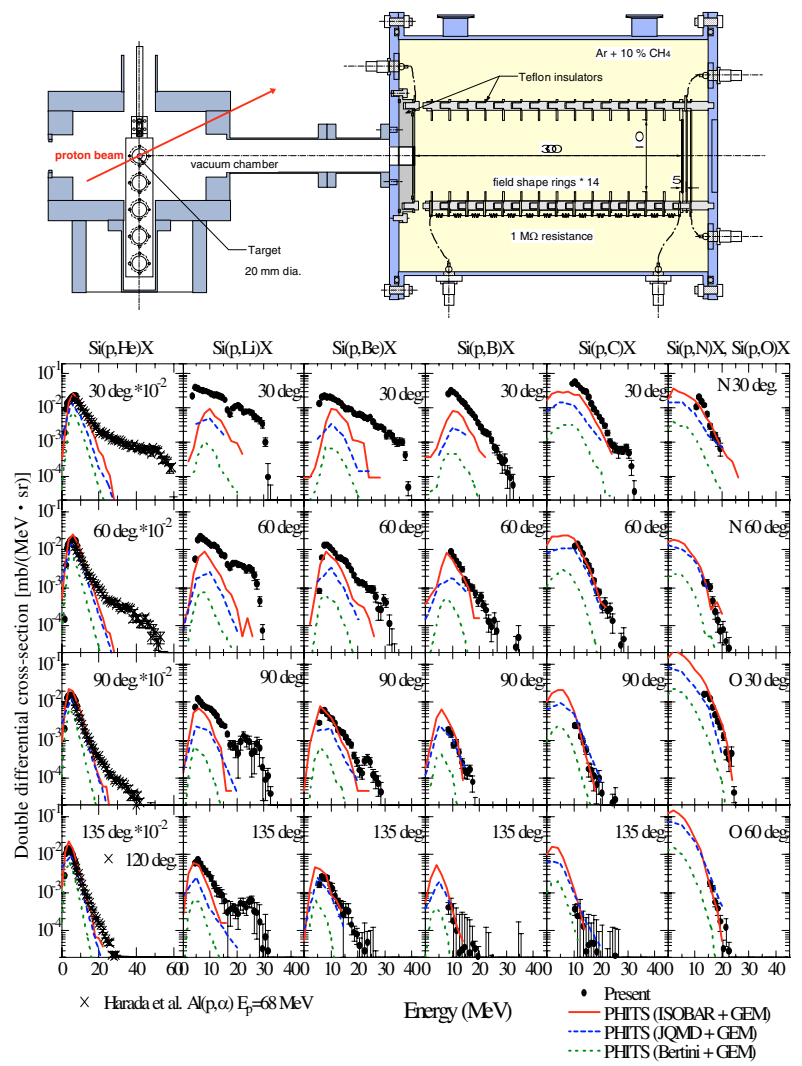

Fig. 15. Bragg curve counter for fragment production studies (upper) and the results of proton induced fragment production from silicon at $70 \mathrm{MeV}$ (lower) [22].

production data, Brag Curve Counter (BCC) [22]. Figure 15 illustrates the schematic view of BCC and the experimental data for proton induced fragment production of $\mathrm{Si}$ at $70 \mathrm{MeV}$. The experimental data are compared with theoretical calculations. The figure indicates general trend of underestimation of calculation which is similar as in the case of helium isotopes in $392 \mathrm{MeV}$ data (fig. 13). The group developed too BCC with a segmented anode which is optimized for neutron induced reaction and obtained first data for neutron induced reactions at $65 \mathrm{MeV}$ [22].

\section{Neutron source development}

Intense and high quality neutron sources are essential for studies of nuclear data. In Japan, intense spallation neutron source is under construction as a part of accelerator complex JPARC (Japan Proton Accelerator Research Complex) at Tokai, as a joint project between JAEA and KEK. Within the project, an intense moderated neutron source will be constructed. Besides, an intense ${ }^{7} \mathrm{Li}(\mathrm{p}, \mathrm{n})$ neutron source was installed at Tohoku University.

\subsection{J-PARC neutron source}

J-PARC consists of injector, $400 \mathrm{MeV}$ and $600 \mathrm{MeV}$ drift tube linacs, $3 \mathrm{GeV}$ synchrotron, and $50 \mathrm{GeV}$ synchrotron. Intense 


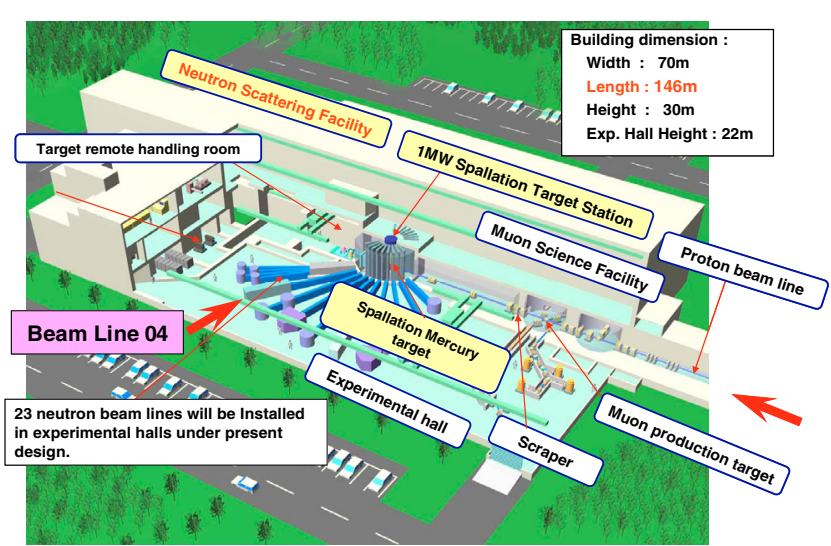

Fig. 16. Layout of Materials and Life Science Facility in J-PARC [23].

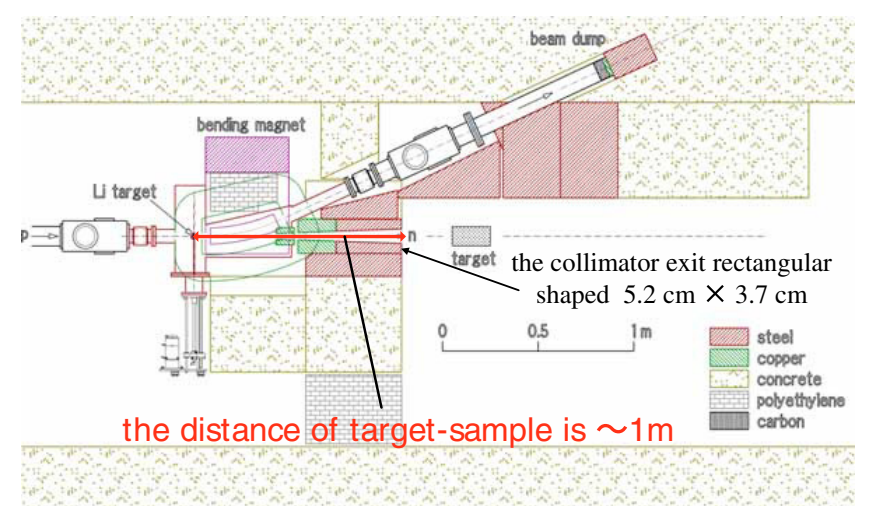

Fig. 17. Layout of intense ${ }^{7} \mathrm{Li}(\mathrm{p}, \mathrm{n})$ neutron source at CYRIC, Tohoku University [21].

beam power of $1 \mathrm{MW}$ is a special feature of the system [23]. A proton beam from $3 \mathrm{GeV}$ synchrotron is used to produce intense neutron beams for research of material and life science through neutron scattering and so on (Material and Life Science Facility: MLF). The $50 \mathrm{GeV}$ beam is for nuclear/particle physics. The $400 / 600 \mathrm{MeV}$ linac will be used for basic research of nuclear transmutation in combination with critical assembly.

Figure 16 illustrates the neutron beam lines in MLF, J-PARC. The beam line No. 4 will be devoted to the neutron physics and nuclear data research using intense moderated beams. It will be operational in 2008. The time-differential neutron beam intensity is very high, then the line is very useful for neutron cross section studies, in particular of samples having very high radioactivity like MA. Under the support of Nuclear Data Project-2, experiments on capture dross section will be started using $4 \pi$ Ge detector developed in Project-1 (sect. 2.4).

\subsection{Intense ${ }^{7} \mathrm{Li}(p, n)$ source}

Figure 17 illustrates a schematic view of the intense ${ }^{7} \mathrm{Li}(\mathrm{p}, \mathrm{n})$ neutron source installed at CYRIC, Tohoku University [24]. The source can provide a neutron flux higher than
$10^{6} \mathrm{n} \mathrm{cm}^{-2} \mathrm{~s}^{-1}$ around $1 \mathrm{~m}$ from the target for $\sim 3 \mu \mathrm{A}$ proton beams and a target of $2 \mathrm{MeV}$ thick. By enabling a short distance between target and irradiation point, this intensity which is the heist level over the world is achieved. The source is used successfully for cross section studies of fragment production and neutron-induced activation, and also for semiconductor irradiation experiment which requires high beam intensity. In this source, neutrons to $\sim 25 \mathrm{deg}$. can also be used for experimental tail correction ${ }^{1}$ by combining with the data at 0-deg [24].

\section{Summary}

Recent progress in nuclear data measurements in Japan is reviewed. Development of experimental techniques and promotion of experiment using the techniques developed is now opening a new area of experiment. In particular, experiments in J-PARC are highly expected to produce a new generation data owing to very high intensity of neutron beam and powerful $4 \pi$ Ge $\gamma$-ray detector.

The author expresses his thanks to Drs H. Harada (JAEA), M. Hagiwara (KEK), M. Igashira (TIT), I. Murata (Osaka University). N. Shigyo and S. Uozumi (Kyusyu University), for kindly providing him with useful information in providing the manuscript.

\section{References}

1. H. Harada et al., J. Nucl. Sci. Technol. 44, 103 (2007).

2. H. Harada et al., Nucl. Instrum. Meth. A 548, 455 (2005) (these proceedings).

3. F. Kitatani et al. (these proceedings).

4. T. Hayakawa et al. (these proceedings).

5. M. Igashira et al. (these proceedings).

6. J. Nishiyama (these proceedings).

7. J. Hori (Kyoto University) (private communication).

8. K. Furutak et al. (these proceedings).

9. K. Kimura et al. (these proceedings).

10. M. Mizumoto et al. (these proceedings).

11. M. Oshima et al. (these proceedings).

12. T. Oishi et al., Proceedings of Science 2006.

13. M. Hagiwara et al., Fusion Sci. Technol. 48, 1320 (2005).

14. S. Kamada et al., AESJ 2006 Fall Meeting.

15. Y. Iwamoto et al., SATIFF Meeting, Pohang, Korea (2006).

16. S. Taniguchi et al., Proc. $10^{\text {th }}$ Symp. On Neutron Dosimetry (to be published in Radiation Protection and Dosimetry).

17. S. Noda et al. (these proceedings).

18. K. Kondo et al. (these proceedings).

19. I. Murata et al. (these proceedings).

20. H. Iwamoto et al. (these proceedings).

21. Y. Uozumi et al. (these proceedings).

22. M. Hagiwara et al., Proc. $10^{\text {th }}$ Symp. Neutron Dosimetry (to be published in Radiation Protection and Dosimetry).

23. http://www.j-parc/

24. M. Baba, Proceedings of Science (2006), Proc. $10^{\text {th }}$ Symp. Neutron Dosimetry (to be published in Radiation Protection and Dosimetry).

\footnotetext{
${ }^{1}$ Correction for backgrounds due to continuum components of the ${ }^{7} \mathrm{Li}(\mathrm{p}, \mathrm{n})$ source [24].
} 